\title{
Estudo das Propriedades de Compósitos Biodegradáveis de Amido/Glúten de Milho/Glicerol Reforçados com Fibras de Sisal
}

\author{
Elisângela Corradini \\ Departamento de Engenharia de Materiais, UFSCar \\ Embrapa Instrumentação Agropecuária, São Carlos/SP \\ José A. M. Agnelli \\ Departamento de Engenharia de Materiais, UFSCar \\ Luís C. de Morais \\ Universidade Federal do Acre-UFAC \\ Luiz H. C. Mattoso \\ Embrapa Instrumentação Agropecuária, São Carlos/SP
}

\begin{abstract}
Resumo: Neste estudo, fibras de sisal foram utilizadas como reforço para a matriz constituída de amido, glúten de milho e glicerol. O teor de fibra em relação a matriz variou de 5 a $30 \%$. O processamento da matriz e dos compósitos foi realizado em um reômetro de torque Haake à $130^{\circ} \mathrm{C}, 50 \mathrm{rpm}$ por 10 minutos. As misturas obtidas foram moldadas por compressão à quente. As propriedades mecânicas; termodinâmico-mecânico (DMTA) e de absorção de água foram investigadas em função do teor de fibras na matriz de amido/glúten de milho/glicerol. O aumento do conteúdo de fibra provocou uma melhoria nas propriedades mecânicas dos compósitos com relação à matriz. O compósito reforçado com $30 \%$ de fibra de sisal apresentou aumento no módulo de elasticidade e tensão na ruptura de aproximadamente de 560 e $162 \%$, respectivamente e diminuição nos valores de elongação na ruptura de $81 \%$. Os resultados obtidos por DMTA mostraram aumento progressivo do módulo de armazenamento (E') e diminuição do módulo de amortecimento (tan $\delta$ ) com o aumento do teor de fibra, confirmando o efeito de reforço das fibras de sisal na matriz de amido/glúten de milho/glicerol. A incorporação das fibras na matriz também provocou diminuição da absorção de umidade e no coeficiente de difusão de água. A análise da morfologia dos compósitos mostrou boa dispersão das fibras na matriz.
\end{abstract}

Palavras-chave: Biocompósito, glúten de milho, amido, glicerol, fibras de sisal.

\section{Study of Properties of Biodegradable Composites of Starch/Gluten/Glycerol Reinforced with Sisal Fibers}

Abstract: Biocomposites using starch/gluten/glycerol as matrix and sisal fibers were produced by melt-mixing in an intensive batch mixer connected to a torque rheometer at $130{ }^{\circ} \mathrm{C}$. The samples were compression molded and than characterized by water absorption and mechanical test. In tensile test, the increasing in the Young's modulus and ultimate tensile strenght were respectively of aproximately 560 and $162 \%$ in relation to matrix, whereas elongation at break decreased. The storage modulus increased with increasing fiber content whereas $\tan \delta$ curves decreased, confirming the reinforcing effect of the fibers. The addition of sisal fibers in matrix decreased the water absorption at equilibrium. The diffusion coefficient decreased sharply with 5\% addition of fiber and further sisal fiber additions provoked only small variation. Optical microscopy observations revealed good dispersion of the sisal fibers into the matrix.

Keywords: Biocomposite, starch, gluten, sisal fibers.

\section{Introdução}

Devido à busca por materiais não agressivos ao meio ambiente, o desenvolvimento de compósitos poliméricos envolvendo o emprego de fibras vegetais e polímeros naturais vêm crescendo cada vez mais ${ }^{[1,2]}$.

As fibras naturais são adicionadas a matriz polimérica visando melhorar suas propriedades e reduzir custos da composição polimérica e/ou a geração de efluentes ${ }^{[3]}$. A utilização das fibras vegetais, tais como juta, algodão, sisal e coco, como reforço em comparação às cargas inorgânicas possui muitas vantagens, tais como: obtenção de materiais de baixa densidade, menor abrasão durante processamento, altos níveis de preenchimento que resultam em aumento na rigidez, elevado módulo específico, aumento na durabilida-

Autor para correspondência: Elisângela Corradini, Embrapa Instrumentação Agropecuária, Caixa Postal 741, CEP: 13560-970, São Carlos, SP, Brasil. E-mail: elisangela@cnpdia.embrapa.br 
$\mathrm{de}^{[4]}$. Além disso, as fibras naturais são biodegradáveis, provenientes de fontes renováveis de grande disponibilidade e de baixo custo ${ }^{[5]}$.

Polímeros naturais tais como: amido e glúten são interessantes matérias primas para serem utilizados como matriz em biocompósitos devido a suas biodegradabilidade e baixo custo $^{[6]}$. Ambos podem ser processados na presença de plastificante, similarmente aos polímeros sintéticos convencionais. Além disso, o amido e o glúten possuem grupos polares que podem interagir com as hidroxilas das fibras lignocelulósicas e assim, resultar em um material com boa propriedade mecânica.

Neste trabalho, fibras de sisal foram utilizadas como reforço para a matriz biodegradável de amido/glúten de milho/glicerol. As propriedades de absorção de água, mecânica e termodinâmico-mecânico e morfologia dos compósitos foram investigadas em função do teor de fibra.

\section{Experimental}

\section{Material}

Os materiais utilizados foram: amido de milho híbrido com aproximadamente $27 \%$ de amilose (nome comercial amisol 3408), glúten de milho $(60-70 \%$ de proteína, $20 \%$ de amido, $4 \%$ de lipídios) foram doados pela Corn Products do Brasil Ltda. Fibras de sisal foram doadas pela UFSCar. Glicerol com grau PA fornecido pela Synth Reagentes Ltda.

\section{Processamento da matriz e dos compósitos}

A composição da matriz amido/glúten de milho/glicerol foi mantida constante na proporção 40/40/20 (\% em peso). Água foi adicionada como um agente de processo na proporção de $10 \%$ em relação a matriz. Foram confeccionados compósitos com teores de fibras de 5, 10, 20 e 30\% em peso em relação à massa total dos polímeros (amido e glúten de milho). As fibras foram moídas em moinho de faca com peneira de 10 mesh.

O processamento da matriz e dos compósitos foi realizado em um reômetro de torque Haake à $130{ }^{\circ} \mathrm{C}, 50 \mathrm{rpm}$ por 10 minutos. As misturas obtidas foram moldadas por compressão à quente numa prensa hidráulica com placas de aquecimento com controle de temperatura. Entre as placas foi colocado o molde constituído de três placas de aço inox de dimensões $(150 \times 120 \times 2,5) \mathrm{mm}$. O material foi transferido para o molde à temperatura de $130^{\circ} \mathrm{C}$, permanecendo sem pressão durante dois minutos para que ocorresse sua fusão parcial e, em seguida foi aplicada uma pressão de 5 ton durante 2 minutos. Posteriormente o molde foi resfriado até atingir $80^{\circ} \mathrm{C}$ e, em seguida, o material moldado foi removido do molde.

\section{Caracterização dos materiais processados}

a) Absorção de água: Os ensaios de absorção de água foram realizados em ambientes com $52 \pm 3 \%$ e $97 \pm 3 \%$ de umidade relativa, obtidas através do uso de soluções saturadas de sais, que foram respectivamente $\mathrm{Mg}\left(\mathrm{NO}_{3}\right)$ e $\mathrm{Na}_{2} \mathrm{SO}_{4}$. $\mathrm{O}$ procedimento de condicionamento foi realizado de acordo com a norma ASTM E $104^{[7]}$. Os corpos de prova foram cortados com um vazador com $11 \mathrm{~mm}$ de diâmetro, a partir de placas moldadas à quente com espessura de aproximadamente de $2 \mathrm{~mm}$. Os materiais foram secos a $105^{\circ} \mathrm{C}$ por 24 horas e foram posteriormente condicionados em recipientes herméticos, contendo as soluções saturadas. As amostras foram pesadas em sucessivos intervalos de tempo até atingir o equilíbrio ou a absorção máxima.

A quantidade de água absorvida (AA) foi calculada pela Equação 1:

$$
A A(\%)=\left(\frac{M_{t}-M_{0}}{M_{0}}\right) \times 100
$$

onde $\mathrm{M}_{0}$ e $\mathrm{M}_{\mathrm{t}}$ são as massas da amostra após um tempo t de exposição à água ou a um ambiente com umidade relativa controlada e antes da exposição do referido ambiente, respectivamente.

O coeficiente de difusão da água foi determinado a partir das medidas de absorção de água a $97 \pm 3 \%$ de umidade relativa, segundo a aproximação de $\mathrm{Crank}^{[9]}$ baseada na Equação 2:

$$
\frac{M_{t}-M_{0}}{M_{\infty}}=\frac{2}{L}\left(\frac{D}{\pi}\right)^{1 / 2} t^{1 / 2}
$$

onde $\mathrm{M}_{\infty}$ é a massa de água absorvida no equilíbrio, $\mathrm{L}$ é a espessura do filme polimérico e $\mathrm{D}$ é o coeficiente de difusão. A Equação 2 é valida somente para curtos períodos de medida, onde $\left(\mathrm{M}_{\mathrm{t}}-\mathrm{M}_{0}\right) / \mathrm{M}_{\infty} \leq 0,5$. O coeficiente de difusão de água foi calculado a partir do coeficiente angular da reta $\left(\mathrm{M}_{\mathrm{t}}-\mathrm{M}_{0}\right) / \mathrm{M}_{\infty}$ em função de $\left(\mathrm{t}^{1 / 2} / \mathrm{L}\right)$ e pode ser expresso como:

$$
D=\frac{(\text { Coef. ang }) \times \pi}{4}
$$

b) Ensaios de tração: Os corpos de prova para os ensaios de tração foram confeccionados de acordo com as dimensões especificadas pela norma ASTM D638M ${ }^{[8]}$, tipo II. Antes de serem submetidos aos ensaios de tração, os corpos de prova foram condicionados a $52 \pm 3 \%$ de umidade relativa e pesados em sucessivos intervalos de tempo até que fosse atingido equilíbrio de absorção. Os ensaios foram realizados numa máquina Instron modelo 5569 com célula de carga de $5 \mathrm{KN}$.

c) Análise termodinâmico-mecânica (DMTA): Os corpos de prova para caracterização termodinâmico-mecânico foram cortados nas dimensões de aproximadamente $(46 \times 1,2 \times 2) \mathrm{mm}$ e submetidos a uma deformação senoidal no modo flexão em três pontos, no intervalo de -100 até $120^{\circ} \mathrm{C}$. As análises foram realizadas em um DMTA Thermal Analyser MKII da Polymer Laboratories. As condições utilizadas foram força dinâmica de 3,0 N, amplitude de $64 \mu \mathrm{m}$, 
freqüência de oscilação de $1 \mathrm{~Hz}$ e taxa de aquecimento de $2{ }^{\circ} \mathrm{C} / \mathrm{min}$.

d) Microscopia óptica: (MO): Filmes dos compósitos, com espessura de aproximadamente $1 \mathrm{~mm}$, foram analisados em um microscópio óptico marca Olympus, modelo CBA. As análises foram realizadas à temperatura ambiente e com aumento de 40x.

\section{Resultados e Discussão}

Os resultados dos experimentos de absorção de água em função do tempo para a matriz de amido/glúten de milho/glicerol e para os compósitos reforçados com 5, 10, 20 e $30 \%$ de fibra de sisal condicionados em ambientes de $52 \pm 3 \%$ e $97 \pm 3 \%$ de umidade relativa, estão apresentados na Figura 1. A matriz apresentou absorção de equilíbrio

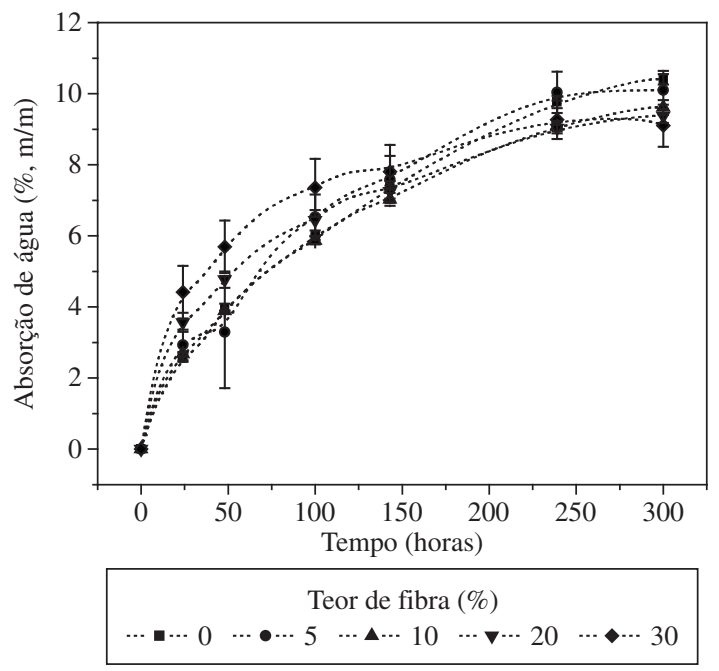

(a)

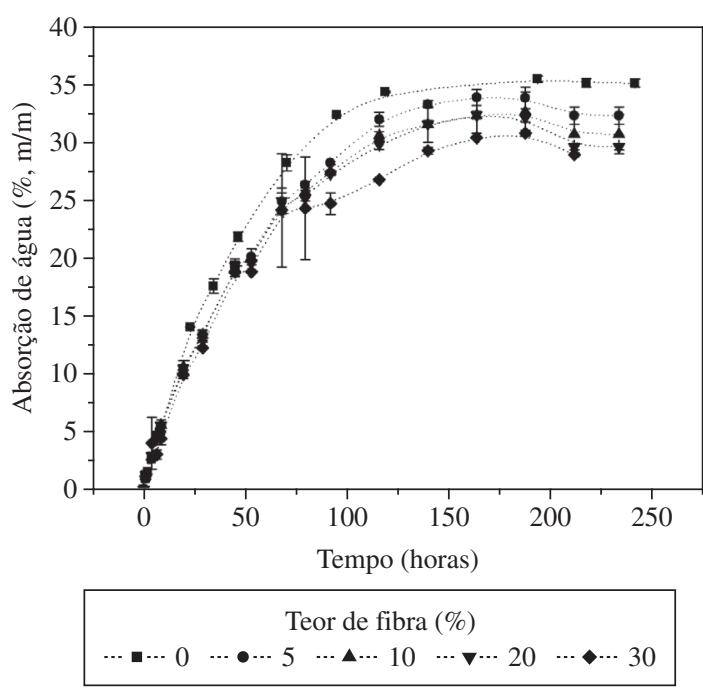

(b)

Figura 1. Absorção de água (\%) em função do teor de fibra para a matriz de amido/glúten de milho/glicerol e para os compósitos preparados com fibras de sisal em ambiente de a) $52 \pm 3 \% \mathrm{RH}$; e b) $97 \pm 3 \% \mathrm{RH}$. em torno de $10,4 \pm 0,2 \%$ à $52 \pm 3 \%$ RH e $35,2 \pm 0,3 \%$ à $97 \pm 3 \%$ RH (Tabela 1), sendo que a adição de $30 \%$ de fibras causou diminuição da absorção de água de equilíbrio de aproximadamente $12,3 \%(52 \pm 3 \% \mathrm{RH})$ e $17,6 \%$ (97 \pm $3 \% \mathrm{RH}$ ) em relação a matriz. A redução da absorção de água com introdução das fibras de sisal é provavelmente devido ao maior caráter hidrofílico da matriz de amido/glúten de milho/ glicerol em relação fibras de sisal.

Na Figura 2 é apresentada a dependência do coeficiente de difusão em função do teor de fibra para os compósitos preparados com fibras de sisal. A matriz de amido/glúten de milho/glicerol apresentou maior coeficiente de difusão, $2,38 \times 10^{-8} \pm 0,47$, sendo que e a adição de apenas $5 \%$ de fibra na matriz reduziu significativamente o coeficiente de difusão e não variou significativamente para teores maiores de fibras. A diminuição do coeficiente nos compósitos com relação a matriz é provavelmente devida a "rede" formada pelas fibras, a qual impede a difusão da umidade através da matriz ${ }^{[10]}$.

Alvarez et al. ${ }^{[1]}$ estudaram as propriedades de absorção de água de compósitos biodegradáveis preparados a partir de uma matriz de MaterBi-Y (uma blenda biodegradável comercial sintetizada a partir do amido, derivados de celulose e aditivos) e fibras de sisal. Os resultados mostraram que a absorção máxima diminui de 28 para $22 \%$ com a introdução de $15 \%$ de fibra na matriz, os valores de coeficiente de difusão

Tabela 1. Absorção de água no equilíbrio em função do teor de fibras para a matriz de amido/glúten de milho/glicerol e para os compósitos com 5, 10, 20 e 30\% de fibras de sisal em ambientes com $52 \pm 3 \% \mathrm{RH}$; e $97 \pm 3 \% \mathrm{RH}$.

\begin{tabular}{|c|c|c|}
\hline \multirow[t]{2}{*}{ Composições } & \multicolumn{2}{|c|}{$\begin{array}{l}\text { Absorção de água no } \\
\text { equilíbrio }(\%, \mathrm{~m} / \mathrm{m})\end{array}$} \\
\hline & $52 \pm 3 \%$ RH & $97 \pm 3 \%$ RH \\
\hline Matriz & $10,41 \pm 0,15$ & $35,16 \pm 0,36$ \\
\hline $\begin{array}{l}\text { Compósito - } 5 \% \\
\text { de fibra de sisal }\end{array}$ & $10,09 \pm 0,54$ & $32,36 \pm 0,73$ \\
\hline $\begin{array}{c}\text { Compósito - } 10 \% \\
\text { de fibra de sisal }\end{array}$ & $9,61 \pm 0,21$ & $30,73 \pm 1,69$ \\
\hline $\begin{array}{l}\text { Compósito - } 20 \% \\
\text { fibra de sisal }\end{array}$ & $9,36 \pm 0,19$ & $29,66 \pm 0,27$ \\
\hline $\begin{array}{l}\text { Compósito - } 30 \% \\
\text { fibra de sisal }\end{array}$ & $9,12 \pm 0,58$ & $28,97 \pm 0,16$ \\
\hline
\end{tabular}

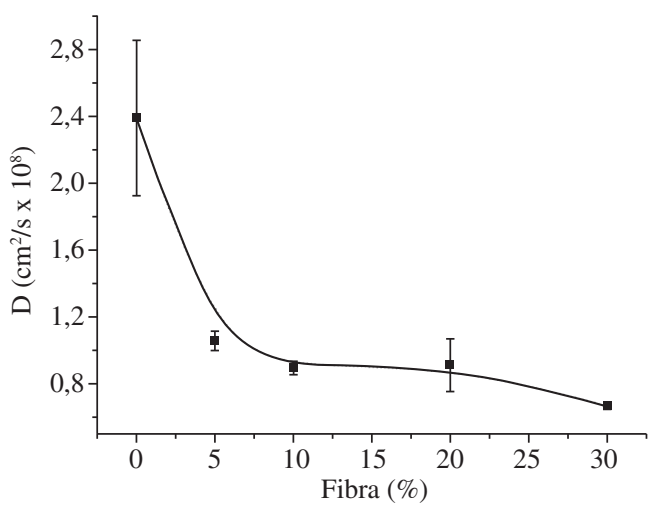

Figura 2. Coeficiente de difusão de água em função do teor fibra para a matriz de amido/glúten de milho/glicerol e para os compósitos reforçados com fibras de sisal. 
foram $9,94 \times 10^{-8} \pm 2,8 \times 10^{-9} \mathrm{e} 8,56 \times 10^{-8} \pm 1,0 \times 10^{-9} \mathrm{~cm}^{2} / \mathrm{s}$ para a matriz e para o compósito com $15 \%$ de fibra de sisal, respectivamente. Comparando esses valores com os resultados apresentados para os compósitos de amido/glúten de milho/glicerol reforçados com fibras de sisal, observa-se que a absorção de água é cerca de $10 \%$ maior que compósitos MaterBi-Y, embora o coeficiente de difusão seja menor. Estas diferenças são em parte devidas às diferenças de constituição química da matriz polimérica e das interações entre água (espécie permeante) e os constituintes da matriz ${ }^{[12]}$.

Na Figura 3 são apresentados os valores de módulo de elasticidade $(E)$, tensão na ruptura $\left(\sigma_{r}\right)$ e elongação na ruptura $\left(\varepsilon_{\mathrm{r}}\right)$ em função do teor de fibra. Como esperado, os valores de E e $\sigma_{\mathrm{r}}$ aumentaram com o aumento do teor de fibras e os valores de $\varepsilon$ diminuíram com a introdução da fibra na matriz. Comparando os valores de $\mathrm{E}, \sigma_{\mathrm{r}}$ e $\varepsilon_{\mathrm{r}}$ obtidos para matriz com os mesmos valores para os compósitos reforçados com $30 \%$ de fibras, observa-se aumento de 560 e $162 \%$ nos valores de E e $\sigma_{\mathrm{b}}$ respectivamente e diminuição de $81 \%$ em $\varepsilon$. Essas variações nas propriedades mecânicas indicam que a incorporação das fibras lignocelulósicas na matriz de amido/glúten de milho/glicerol resultou em forte interação entre a matriz e as fibras.

Wu et al. ${ }^{[13]}$ verificaram um aumento na tensão de ruptura de $20 \%$ com a introdução de $30 \%$ de pó de madeira em uma matriz de glúten plasticificada com glicerol, enquanto que o aumento de tensão na ruptura foi em torno de $162 \%$ para os compósitos reforçados com $30 \%$ fibras de sisal. Isto mostra que as fibras apresentam uma eficiência maior como reforço do que o pó de madeira para este tipo de matriz.

Comparando os valores de E obtidos para o sistema em estudo com os mesmos valores observados para outros compósitos biodegradáveis produzidos a partir de proteínas e amido, descritos na literatura, observa-se que valores observados são próximos e algumas vezes maiores ${ }^{[14]}$. Por exemplo, os valores de módulo observado para os compósitos de amido/glúten de milho/glicerol reforçados com 10 e $30 \%$ de fibra de sisal foram próximos próximos de 300 e $1500 \mathrm{MPa}$, respectivamente, enquanto que o compósito de amido termoplástico reforçado com $15 \%$ de polpa de madeira (Kraft $)^{[15]}$, apresentou valor de E máximo em torno de $341 \mathrm{MPa}$ e os compósitos de PCL/amido reforçado com fibras de sisal mostraram valores máximos de $\mathrm{E}$ de $687 \pm 119 \mathrm{MPa}^{[16]}$.

Curvas de DMTA para a matriz de amido/glúten de milho/glicerol e para os compósitos reforçados com fibras de sisal são apresentados na Figura 4. A mistura de amido/glúten de milho/glicerol exibiu três transições. A primeira é atribuída ao glicerol ${ }^{[17]}$. A segunda transição é referente à Tg do amido, a qual é dependente do teor de plastificante ${ }^{[18]}$. Corradini et al. ${ }^{[19]}$ mostraram que $\mathrm{Tg}$ do amido diminui de $32{ }^{\circ} \mathrm{C}$ à $-4{ }^{\circ} \mathrm{C}$ quando o teor de glicerol aumentou de 22 para $40 \%$. A terceira transição, de acordo com a literatura ${ }^{[13,20]}$, é referente a zeína (proteína presente no glúten de milho). A presença de duas transições indica que a matriz de amido/glúten de milho/glicerol é um sistema imiscível. A introdução da fibra na matriz causou um aumento progressivo no módulo de armazenamento devido à restrição dos segmentos de cadeias poliméricas provocados pelo reforço. Observa-se também a diminuição na magnitude de Tan $\delta$ nos compósitos com relação a matriz. Isto indica a existência de boa adesão entre as fibras lignocelulósicas e a matriz de amido/glúten de milho/glicerol. Em situações onde não há boa adesão entre a fibra e a matriz o movimento de fricção entre a matriz e a

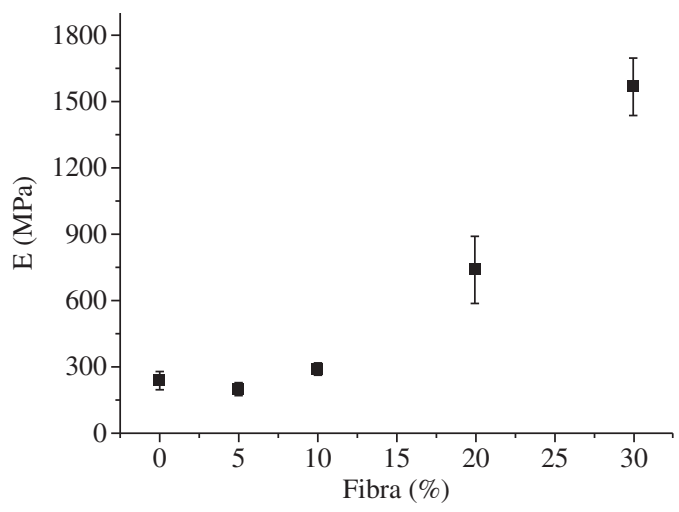

(a)

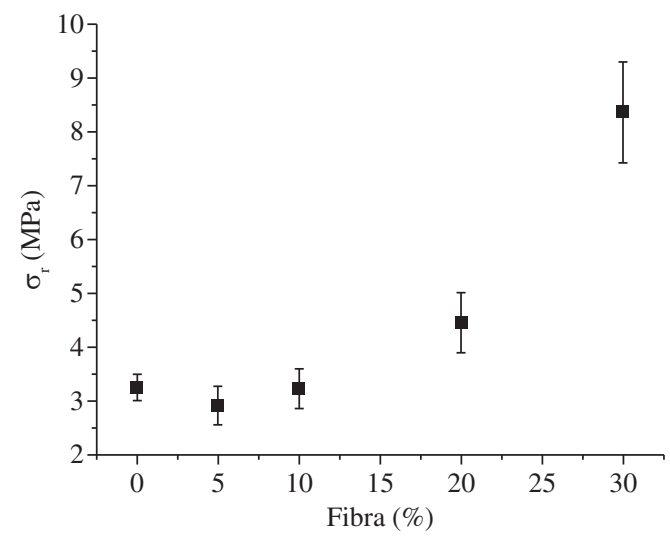

(b)

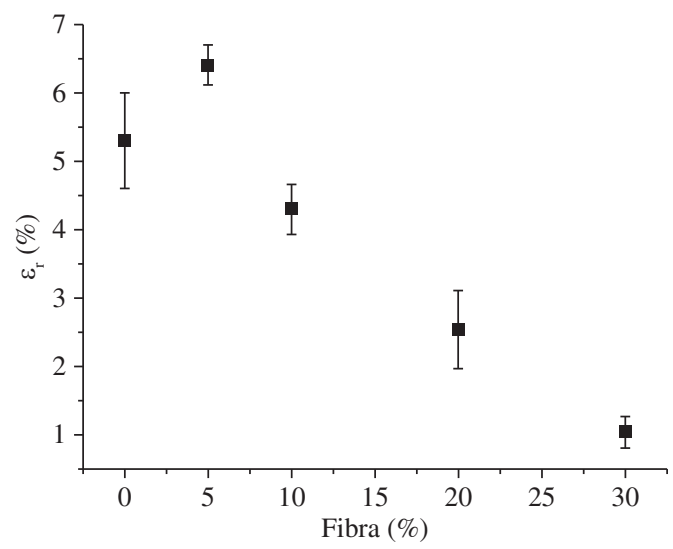

(c)

Figura 3. a) Módulo de elasticidade (E); b) tensão na ruptura; e c) elongação na ruptura em função em função do teor de fibra (\% massa/massa) para os compósitos de amido/glúten de milho/glicerol reforçados com fibras de sisal. 
fibra dissipa energia podendo causar um aumento nos valores de $\operatorname{Tan} \delta$.

Na Figura 5 são apresentadas as fotos obtidas por microscopia óptica dos compósitos reforçados com diferentes teores de fibra de sisal. Observa-se em geral, uma boa dispersão das

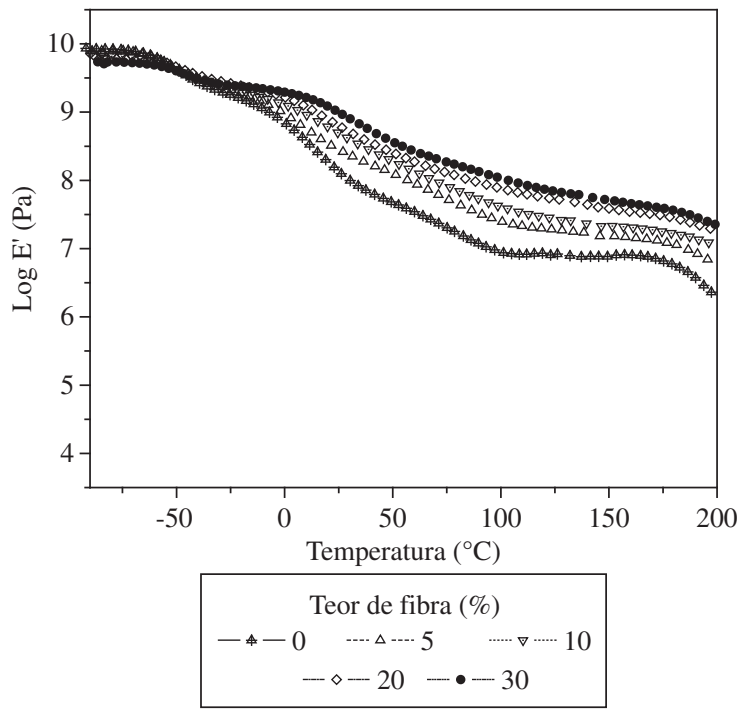

(a) fibras de sisal na matriz. No entanto, as fibras são de tamanhos diferentes e a distribuição das fibras dentro da matriz é aleatória. Observa-se ainda que as fibras foram desfibriladas durante processamento, o que pode aumentar a adesão entre a fibra de sisal e a matriz.

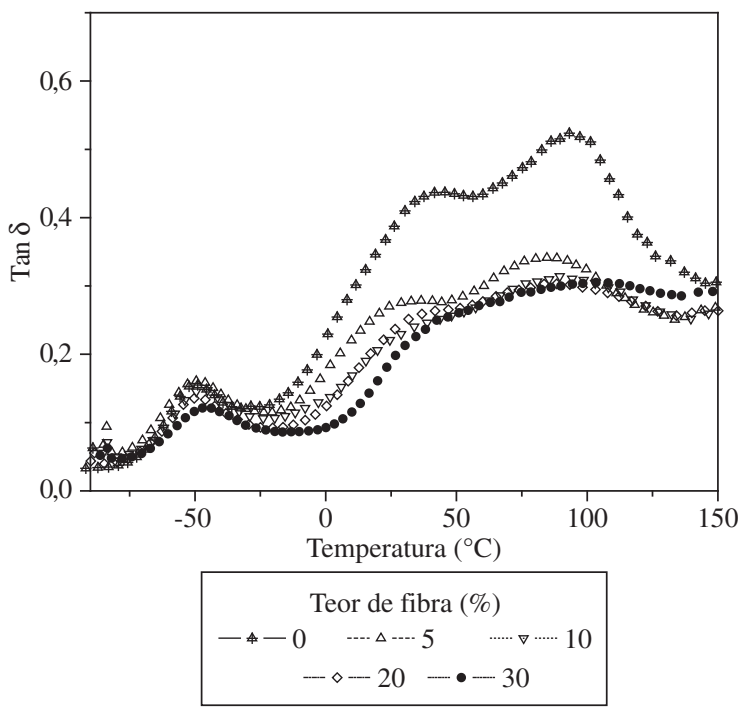

(b)

Figura 4. Curvas de DMTA para a matriz de amido/glúten de milho/glicerol e para os compósitos reforçados com fibras de sisal. a) módulo de armazenamento; e b) amortecimento $(\tan \delta)$.

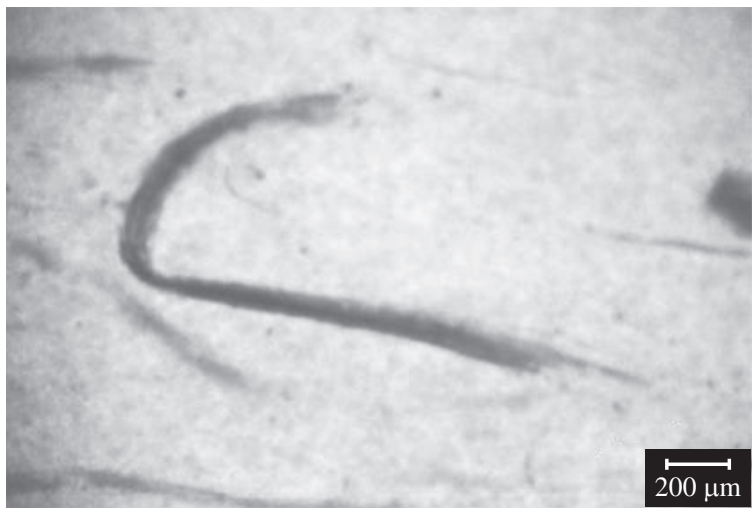

(a)

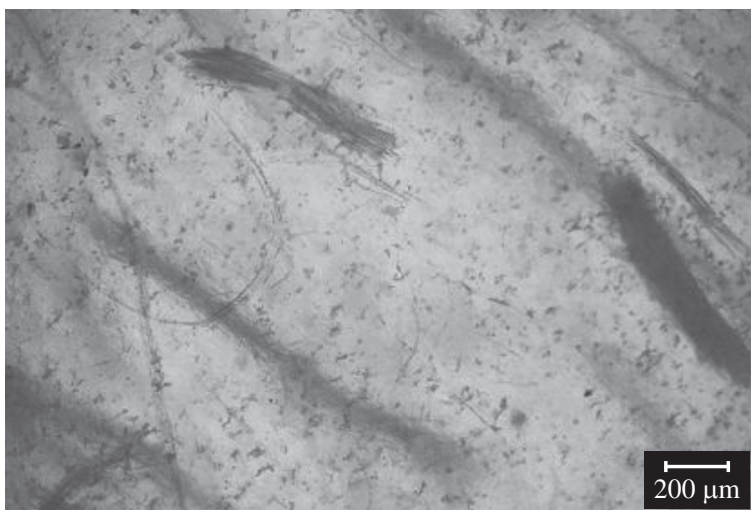

(b)

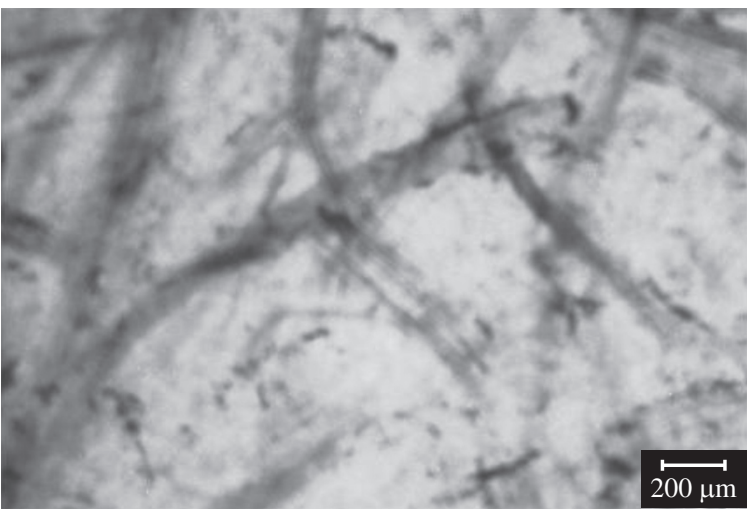

(c)

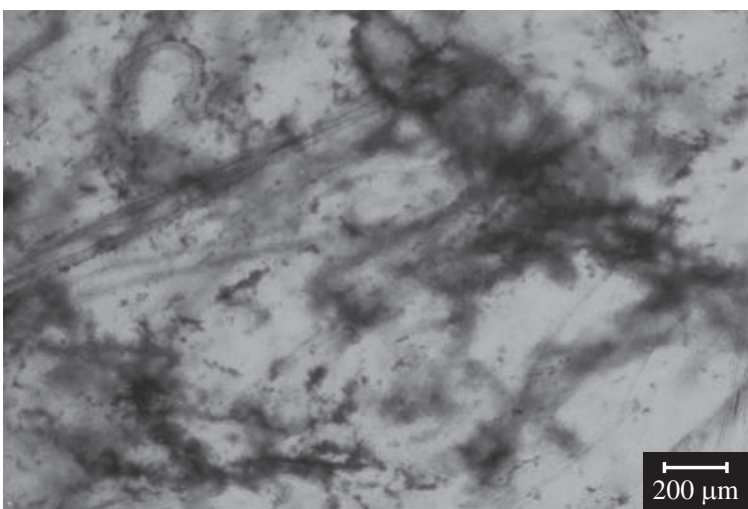

(d)

Figura 5. a) Fotos obtidas por microscopia óptica para os compósitos de amido/glúten de milho/glicerol reforçados com 5\% de fibra de sisal; b) $10 \%$ de fibra de sisal; c) $20 \%$ de fibra de sisal; e d) $30 \%$ de fibra de sisal. 


\section{Conclusões}

Os resultados indicam que as fibras de sisal apresentam um grande potencial para reforçar a matriz de amido/glúten de milho/glicerol. A absorção de água diminui com a introdução da fibra na matriz e o coeficiente de difusão sofreu uma diminuição acentuada com a adição de apenas $5 \%$ de fibra e permaneceu praticamente constante para maiores teores de fibra. As fibras apresentaram uma boa dispersão dentro da matriz. A incorporação da fibra na matriz favoreceu a uma melhora nas propriedades mecânicas dos compósitos com relação à matriz. $\mathrm{O}$ aumento do conteúdo de fibra provocou aumento no módulo de elasticidade e resistência à tração e diminuição da deformação. O módulo de armazenamento (E') aumentou com o aumento do teor de fibra enquanto o amortecimento $(\tan \delta$ ) diminui, confirmando o efeito de reforço das fibras de sisal.

\section{Agradecimentos}

Os autores agradecem à Corn Products do Brasil pela doação das amostras de amido e glúten de milho, ao DEMA/ UFSCar, à FAPESP, CNPq e à Embrapa/CNPDIA pelo apoio técnico e financeiro.

\section{Referências Bibliográficas}

1. Zhang, X. Q.; Do, M. D.; Dean, K.; Hoobin, P. \& Burgar, I. M. - Biomacrom., 8, p.345, (2007).

2. Bhatnagar, A. \& Sain, M. - J. Reinf. Plast. Compos., 24, p.1259, (2005).

3. Leão, A. L.; Carashi, J. C.; Tan I. H.; Kozlowski, R. \& Manys, S. - Plast. Ind., 31, p.214, (2001).

4. Samir, M. A. S. A.; Alloin, F.; Sanches, J. Y. \& Dufresne, A.- Polymer, 45, p.4149, (2004).

5. Mohanty, A. K.; Misra, M. \& Drzal, L. T. - J. Polym. Environ., 10, p.19, (2002).
6. Yu, L.; Dean, K. \& Li, L. - Prog. Polym. Sci., 31, p.576, (2006).

7. ASTM D-638M-96 - "Standard test for tensile properties of plastics", Philadelphia p.11, (1996).

8. ASTM E-104-85 - "Standart pratice for maintaining constant humidity by means of aqueous solutions", Philadelphia, p.11, (1996).

9. Crank, J. - "The Mathematics of Diffusion”, Clarendon Press, Oxford, (1975).

10. Anglès, M. N. \& Dufresne, A. - Macromolecules, 33, p.8344, (2000).

11. Alvarez, V. A.; Ruscekaite, R. A. \& Vázquez, A. - J. Compos. Mater., 37, p.1575, (2003).

12. Stancell, A. F. - "Diffusion through polymers", in: Polymer Science and Materials, cap.12, Tobolsky, A.V.; Mark, H. F (eds). John Willey \& Sons, New York, (1985).

13. Wu, Q. X.; Sakabe, H. \& Isobe, S. - Ind. Eng. Chem. Res., 42, p.6765, (2003).

14. Briassoulis, D. - J. Polym. Environ. 12, p.65, (2004).

15. Carvalho, A. J. F.; Curvelo, A. A. S. \& Agnelli, J. A. M. - Int. J. Polym. Mater., 51, p.647, (2002).

16. Cyras, V. P.; Iannace, S.; Kenny, J. M. \& Vázquez, A. Polym. Compos., 22, p.104, (2001).

17. Lourdin, D.; Bizot, H. \& Colonna, P. J. - Appl. Polym. Sci., 63, p.1047, (1997).

18. van Soest, J. J. G. \& Vliegenthart, J. F. G. - Focus, 15, p.208, (1997).

19. Corradini, E.; de Medeiros E. S.; Carvalho, A. J. F.; Curvelo, A. A. S. \& Mattoso, L. H. C. - J. Appl. Polym. Sci., 101, p.4133, (2006).

20. Lawton, J. W. - Cereal Chem., 69, p.351, (1992).

Enviado: $30 / 11 / 07$

Reenviado: $12 / 05 / 08$

Aceito: $11 / 07 / 08$ 